\title{
Tinea incognito: Report of two cases
}

\author{
S. K. Kedia ${ }^{1}$ M. Mathur ${ }^{2}$
}

Senior Resident ${ }^{1}$ Asso. Professor ${ }^{2}$ Department of Dermatology, College of Medical Sciences, Bhartpur, Chitwan district, Nepal.

\begin{abstract}
Tinea incognito ${ }^{1}$ remains diagnostic challenge even to expert specialist because of modified clinical presentation caused by of inappropriate use of topical steroid. Topical steroid are being prescribed frequently for vary many dermatosis including fungal infection of skin and being used by patient as "over the counter drug" leading to varied clinical presentation of common dermatosis. This case report is of iatrogenic dermatosis that was invariably misdiagnosed by general physicians.
\end{abstract}

Key words: Tinea incognito, dermatosis, fungal infection.

\section{Introduction}

Dermatophytes are a group of fungi that can infect the skin, hair, and nails because of their ability to use keratin. Skin lesions begin as flat scaly plaques with raised border that extends in all directions at varying rates. This "advancing” scaly borderoften contains red papules or vesicles. As the border progresses outward, the central area becomes brown or hypo pigmented and less scaly. There might be one or more annular lesions, which could be pruritic of varied degree. ${ }^{2}$

Tinea incognito ${ }^{1}$ is the name given to a dermatophyte infection treated with topical or oral corticosteroids, which then loses some of its classical characteristic features. Corticosteroids decrease inflammation and give a false impression that the rash is improving, while dermatophytes flourish because of immune suppression. Lesions may look different, scaling and a well-defined border might be absent. The skin lesion may greatly expanded to demonstrate Correspondences: Dr. S.K. Kedia

E-mail: sima_kedia@yahoo.com diffuse erythema, telangiectasia, scattered papules, pustules, and hyperpigmentation. Tinea incognito is most often seen on the groin, face, and dorsal aspect of the hand. Skin lesions do not have classical morphology. Disease may remain challenge to general practicerners or at times misdiagnosed by experienced clinicians culminating further treatment with topical steroid. We report two cases suffering from tinea infection at two different sites, under diagnosed and received topical steroid treatment for the disease.

\section{Case no 1.}

A 63-year old female farmer, known case of hypertension and chronic obstructive pulmonary disease for last 4 years and was on various medications. She was smoker, smokes 5 packs per year, non alcoholic. She developed erythematous plaque lesions with fine scaling on bilateral forearm and at left malar region. She was prescribed topical clobetasol cream by general physician and within few days of application 
of cream, she noticed papules and nodules with scaling at the periphery of the plaques. She was referred to dermatology department for consultation.

On examination, she was found to have erythematous plaque with papules, nodules and fine scaling at forearm on both side (Fig. 1) and erythematous papulo nodular lesion at left malar region (Fig. 2). No other skin lesion was noticed over body. T. incognito was diagnosed and skin scrapings were obtained. Potassium hydroxide mount of skin scraping showed multiple branched septate hyphae. Trichophyton verrucosum was isolated on culture. Routine blood investigations were within normal limits.

\section{Case no 2.}

A 35-year old male office worker, attended outpatient clinic with itchy erythematous lesions at thighs bilaterally for one month. He applied corticosteroid antibiotic combination cream prescribed by general physician. Two weeks later, he noticed multiple erythematous plaques with vesicular scaly at periphery of lesions. Skin lesions did not improve. He was non smoker, non alcoholic and had no any systemic illness.

On examination, he was found to have multiple erythematous papulo-vesicular lesions and plaques of different size bilaterally over medial aspect of thighs (Fig. 3). Diagnosis of T. incognito was made. $\mathrm{KOH}$ mount of skin scrapping showed multiple hyphae (Fig.4). Epidermophytone floccosum was grown on culture. Routine investigations were within normal limits.

\section{Discussion}

Ive and Marks ${ }^{1}$ in 1968 coined the term 'tinea incognito' for cases of dermatomycosis, erroneously treated with topical steroids, having clinical manifestations that mimicked other skin conditions, such as seborrhoic dermatitis, folliculitis, rosacea, eczema. Only one third of cases have typical 'ringworm' lesions. T. incognito is difficult to diagnose clinically because of the absence of the typical 'ringworm' appearance i.e. concentric erythematous rings with scaling. Both of our patients were under diagnosed and high potent topical steroid had been prescribed by physicians.

Marks in 1978, stressed that steroids reduce cellmediated inflammatory response, enable the infection to spread and modify clinical manifestations. Although clinical manifestations of T. incognito may vary widely, the prevalent one are erythematous plaques with papules and nodules but skin lesions mimicking eczema, pityriasis-rosea, atopic dermatitis, contact dermatitis and resembling the eczema associated with venous insufficiency. Diagnosis may be difficult in pyodermalike forms of tinea incognito which is caused by zoophilic species and associated with severe inflammation. Only mycological examination can provide the correct diagnosis in this situations. ${ }^{4}$

Dermatological therapy has been greatly helped in recent years by the introduction of a variety of potent therapeutic agents such as corticosteroid and various immunosuppressive agents. The diseases are easier to control but it has also resulted in an increase of iatrogenic disease by misuse of these drugs. These agents are misused for the treatment of common dermatosis as in our cases or even by qualified specialist particularly when diseases are under diagnosed..$^{5}$ The clinical appearance of common skin diseases may be rendered almost unrecognizable by use of topical steroids particularly by the use of fluorinated derivatives. ${ }^{6}$ It is surprising to find that corticosteroids are frequently used in the management of dermatosis even for infective skin disease which is of great concern. 
The undoubted ability of steroids in relief of symptoms by the suppression of inflammation and patient satisfaction is also assured by the treatment. Therefore topical and or systemic steroid are frequently prescribed by practicerners. . The lesions relapsed once the treatment is stopped. Both of our cases applied corticosteroid cream topically that changed the classical clinical presentation. Diagnosis of T. incognito was suspected and confirmed by $\mathrm{KOH}$ mount which revealed the presence of translucent, branching septate hyphae.

In the first case described by Ive \& Marks, ${ }^{1}$ dermatophytes isolated were T. rubrum and E. floccosum however Mentagrophytes, M. canis,

Figure.1 showing erythematous plaques with papules and nodule bilatally of forearm

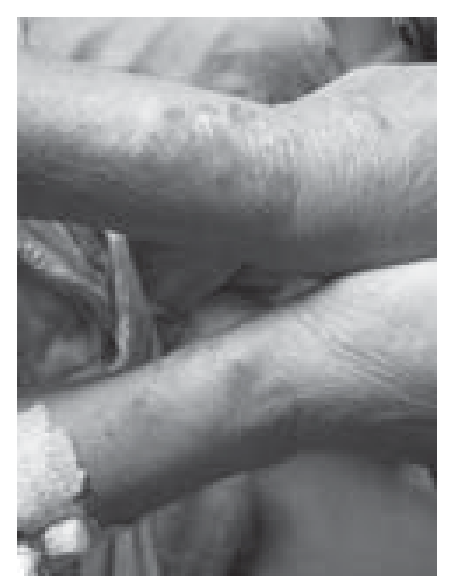

Figure.2 Showing erythematous plaques with papules and nodule on malar region.

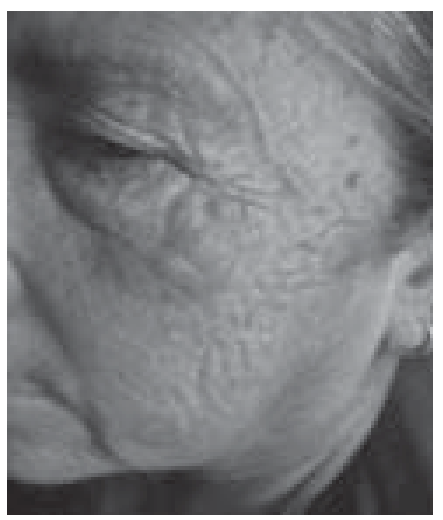

T. violaceum, M. Gypseum and Trichophyton tonsurans species were also reported. ${ }^{7,8}$ In skin sample of our first case, $T$. verrucosum was isolated and in second case E. floccosum.

Treatment includes the immediate cessation of all steroid medication. Patients should be warned for transient flaring of the lesion by use of steroid. Implementation of an appropriate topical and or systemic antifungal regimen may cure the disease. Patients should be advised not to reinstitute the steroids. Our patients relieved of disease after prescribing topical and systemic antifungal treatment.

Figure.3 Showing multiple erythematous papulovasicular lesions and plaques over medial aspect of thigh.

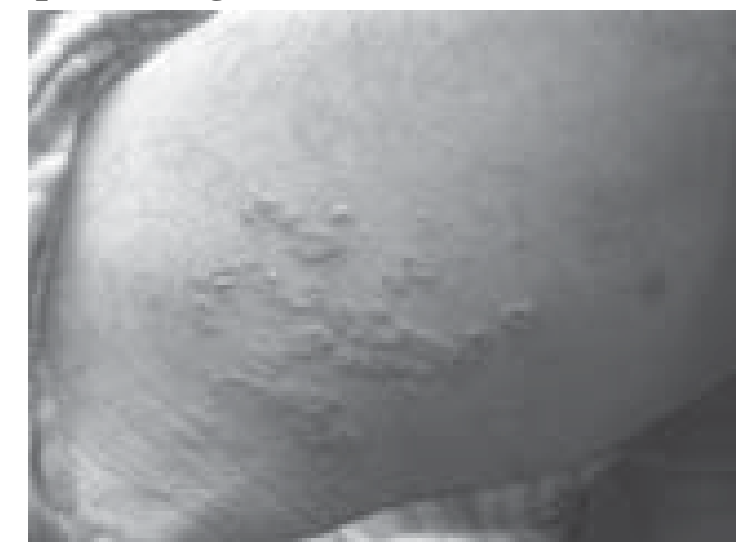

Figure.4 Showing fungal hyphae in KOHmount (40x)

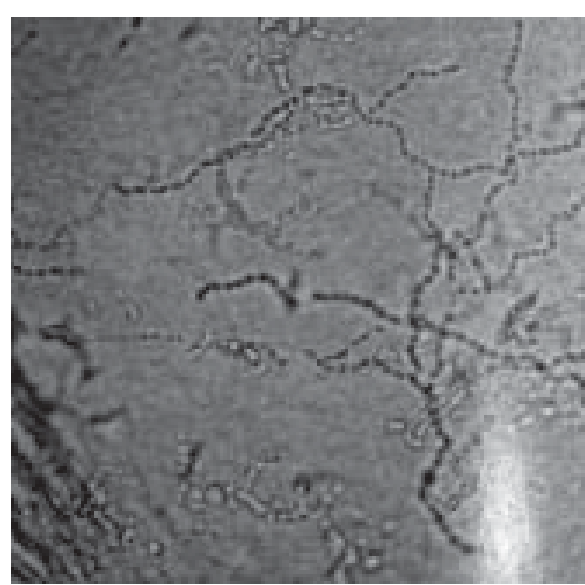


S. K. Kedia et al, Tinea incognito: Report of two cases

\section{References}

1. F.A.Ive, R. Marks . Tinea incognito. Br Med J 1968; 3: 149-52.2

2. E.B.Smith, E.H.Tschen. Superficial fungal infections: Clinical diagnosis and laboratory confirmation. Consultant 1982; 108-3

3. R. Marks Tinea incognito. Int J Dermatol 1978;17: 301-2.

4. M.L. Elgart Tinea incognito: an update on Majocchi granuloma. Dermatol Clin 1996; 14: 51-5

5. H.C. Korting, M.J.Kersher, M. Schafer-Korting.Topical glucocorticoids with improved benefit/risk ration:do thay exist?J AmAcad dermatol 1992;27(1):87-92
6. L.A. Drake, S.M. Dinehart, Guidelines of care for the use of topical glucocorticoids, J Am Acad Dermatol 1996;35(4): 615-9

7. J. Faergemann, T. Fredriksson, O. Herczka et al. Tinea incognito as a source of an "epidemic" of Trichophyton violaceum infections in a dermatologic ward. Int $J$ Dermatol 1983; 22: 3940.

8. C. Romano, F. Asta, L. Massai Tinea incognito due to Microsporum gypseum in three children. Pediatr Dermatol 2000; 17: 141-4. 